\title{
Contexts and Mechanisms That May Facilitate or Inhibit the Implementation of Assisted Decision- Making Policy With Older People in Acute Care Settings: A Qualitative Analysis of the Experiences of Health and Social Care Professionals, Older People and Family Carers.
}

Deirdre O'Donnell ( $\square$ deirdre.odonnell@ucd.ie )

University College Dublin

Carmel Davies

University College Dublin

Lauren Christophers

University College Dublin

Éidín Ní Shé

UNSW Sydney

Sarah Donnelly

University College Dublin

Thilo Kroll

University College Dublin

Research Article

Keywords: Health decision-making, supported decision-making, older people, acute care, dementia, capacity, lived experience, qualitative

Posted Date: February 22nd, 2021

DOl: https://doi.org/10.21203/rs.3.rs-211030/v1

License: (1) This work is licensed under a Creative Commons Attribution 4.0 International License.

Read Full License 


\section{Abstract}

Background: Assisted Decision-Making (ADM) legislation in Ireland provides a statutory basis for the right of all individuals, including those with a disability, to be supported to participate as fully as possible in all decisions which affect their lives. ADM with older people who have impaired cognition may prove challenging for acute healthcare services.

Methods: A qualitative exploration of the experiences of health and social care professionals, older people with and without a diagnosis of dementia and their family carers $(\mathrm{N}=39)$ was conducted. A realist programme theory described by Davies et al.(2019) provided an initial coding framework which was adapted through inductive coding of the accounts.

Results: Personalisation was established as the core theme, supported by four additional themes reflecting implementation domains: Environment and Resources, Social Restructuring, Education, Training and Enablement and Culture and Leadership.

Conclusions: A restructuring of the social and physical infrastructure of acute settings was found to be necessary to maximise insight, ascertain preferences and assist the decision-making of older people. A cultural climate which fosters personalisation is required alongside the cultivation of risk tolerance, collective leadership and inter-professional collaboration. The adapted programme theory described in this article informs policy and practice planning in Ireland. It also contributes to wider debates internationally on the role of socio-cultural and political contexts for ADM implementation.

Patient or Public Contribution: A panel of public representatives of older people were consulted in the development of the grant application. A representative from Alzheimer's Society Ireland and Family Carers Ireland were project steering committee members guiding design and strategy.

\section{Background}

The UN Convention on the Rights of Persons with Disabilities provides an international legislative basis for the human rights of all persons, including those with a disability, to participate as fully as possible in all decisions which affect their lives, including health and social care (United Nations General Assembly, 2007). Pursuant to the convention, international policy and legislative advances have focused on a human-rights based approach to supporting all individuals to make decisions (Boyle, 2011; Griffith and Tengnah, 2008; James and Watts, 2014; Livingston et al., 2010). There has been a particular focus on supporting those with a disability, including those with impaired cognitive capacity, to exercise their legal capacity on an equal basis with others (Knight et al., 2018).

It's been argued that a rights-based approach to healthcare decision-making is necessary to support people in exercising their citizenship and making choices about their health and social care (Devi, 2013; McDonald, 2010; Phelan and Rickard-Clarke, 2020). Within healthcare, a rights-based approach to decision-making represents a powerful alternative to the traditional "best interests" approach involving an 
objective assessment of needs (Donnelly, 2019; Giampieri, 2012). A rights-based approach to healthcare provides a socio-political dimension because it is concerned with examining power. It extends the principles of person-centredness towards the empowerment of individuals to exercise their autonomy concerning decisions which affect their lives.

Assisted Decision-Making (ADM) or Supported Decision-Making is a central tenet of a human-rights approach. A guiding principle of ADM is the presumption that all individuals have decision-making capacity and this capacity should be maximised through different levels of support appropriate to different levels of decision-making capacity. This may include help with accessing information, giving explanations in a manner the individual can understand, communicating the decision or acting to implement the decision on the individual's behalf (Devi et al., 2011). This may also involve the strategic use of decision supporters who can provide insight into an individual's will and preferences and assist with the communication of information between the individual and health and social care professionals (HSCPs). ADM focuses attention on how people with disabilities, including cognitive impairments, can be supported to make decisions about their health and social care on an equal basis with others.

Within the Irish health system, The Assisted Decision-Making (ADM) (Capacity) Act (2015) offers HSCPs a legal framework to support the decision making of patients and clients; ensuring that their will and preference are at the centre of those decisions (Kelly, 2017; O'Donnell et al., 2018). This legislation places an onus on HSCPs as well as decision supporters to maximise the capacity of individuals to make decisions which affect their lives. Translating this legal framework for ADM into the social and cultural contexts of health and social care practice will be challenging and requires a nuanced understanding of complex implementation factors including social environment as well as leadership, resourcing and education of HSCPS and the general public.

A rapid realist review (RRR) of international evidence has identified key challenges to implementing ADM into health and social care practices (Davies et al., 2019). The RRR identified from the literature four key domains presented as an initial programme theory (See Figure One). These domains include the preparation of HSCPs and the public to enable assisted decision-making by providing the supports required to maximise a person's capacity to make decisions. They also encompass the cultural, environmental and social contexts in which healthcare is organised and which may require adaptation to assist decision-making. The review highlighted that the promotion of ADM is more likely to be successful in socio-cultural contexts that embed ADM as a core component of person-centred care (See for example McCormack et al. (2017)).

Implementation of ADM legislation is likely to be particularly challenging to the organisation of acute care services for older people with fluctuating or cognitive impairments (O'Donnell et al., 2018). Older person care is complex as disease types, progression and symptom profile vary over time while social needs, socio-economic status and available resources also play a role (Bridges et al., 2019; McGilton et al., 2018). Ireland has an aging population with $19.1 \%$ of the overall population aged 65 or older, according to recent data $(C S O, 2017)$. This has significant implications for the acute hospital setting, 
where up to $25 \%$ of older patients have dementia, rising to $40 \%$ for patients aged over 70 (Timmons et al., 2015). Older people with dementia often have complex needs, fluctuating capacity and require support for multi-faceted decision making (Timmons et al., 2015). Much of this population have multiple chronic conditions necessitating an integrated care pathway from primary to acute with coordination between services. Fragmented systems and care can lead to further stress and burden for patients, caregivers and HSCPs (McGilton et al., 2018). This population are also highly vulnerable to abuse and neglect, with up to $15.7 \%$ of people over 65 having experienced abuse in some form (Yon et al., 2017).

Balancing the empowerment of older people alongside their protection and complex needs in a busy setting of an acute hospital is a major challenge for ADM implementation. This will require consideration given to how staff are prepared to engage in ADM as well as the sociological, contextual and environmental factors involved in supporting the decision-making of older adult patients in acute care. The current study aims to expand our understanding of the dynamics of healthcare decision-making with older people in an acute environment. The realist Programme Theory described by Davies et al. (2019) provides an initial coding framework which was adapted through inductive coding of the narrative accounts of HSCPs, older people and family carers making decisions in an acute care setting in Ireland. The modified programme theory presented in this article supports responsive and context sensitive implementation of ADM with older people in acute health care settings.

\section{Methods}

This is a cross-sectional, exploratory, qualitative study which used narrative interviews to explore the lived experiences of older people, family carers and HSCPs reflecting upon decision-making in acute care settings.

\subsection{Sample and Recruitment}

The study participants were HSCPs working in the acute care of older people, older people (including those with a diagnosis of dementia), and family carers (see tables 1-4). It was a convenience sample drawn from two large urban academic teaching hospitals in Ireland. Project steering committee collaborators, who are health care professionals from each of the hospitals (three consultant geriatricians, one advanced nurse practitioner and the end-of-life care coordinator), acted as a link between the research interview team (DOD, ENS, SD and FF) and potential participants, facilitating recruitment. The research team attended multi-disciplinary care-of-the-older-person team meetings and journal club sessions in the two sites and presented on the study. HSCPs who expressed interest were emailed a participant information sheet and invited to participate.

Convenience sampling was also used to recruit older people, including those with a diagnosis of dementia, and family carers . Recruitment was facilitated via our public and patient representative organisations Family Carers Ireland, the Alzheimer's Society of Ireland and SAGE Advocacy. Each organisation promoted the study via different forums (member meetings, emails, newsletters, social 
media, text message and via word of mouth). Inclusion criteria were older people (self-identified) who had a recent experience of an acute care admission or family carers who had a recent experience accompanying older relative/friend to the hospital. Once participants indicated their willingness to take part in the research, they were provided with a consent form to read in their own time and share with a nominated decision-making assistant. They were offered the opportunity to have a discussion with a member of the research team as well as their designated decision-making assistant at any point prior to or while giving consent to participate.

\subsection{Data generation}

Interviews took place between January and June 2019. Interviews with HSCPs took place in the respective clinical locations, while the older people and family carers were interviewed at home or at an alternative place of their choice. We used semi-structured interview guides. The interviewees were asked to narrate a recent acute care experience with follow-up questions eliciting further narrative reflection as well as an explanation concerning what is required to embed ADM into acute care. The Alzheimer's Society of Ireland advised that some older people with a diagnosis of dementia may have challenges in narrating a personal experience. With their guidance we created a scenario as a prompt if required by interviewees. Only one interviewee required this innovation. All interviews were audio-recorded with permission and subsequently transcribed.

\subsection{Analysis}

A framework analytical approach (Smith and Firth, 2011) was adopted for this study. Inductive thematic coding of the data were undertaken via a licensed version of NVivo 12 Pro. We used a two-step process: (1) initial open vivo coding (2) aggregation of vivo codes and abstraction into higher-order themes. All the interviews were coded into the lower order vivo codes by one researcher (DOD). These were then verified by a second researcher (LC). Discrepancies between the two researchers were discussed and resolved at weekly meetings with a third researcher (CD). At these meetings the second order thematic clusters (step two) were agreed through consensus. At this point it was felt that data saturation had been achieved as the variation in the data coded into the thematic clusters levelled off.

Presentations of initial thematic findings and a tentative thematic structure were made to each of the participant cohorts. Journal club sessions were run in each of the hospital sites and participants were invited to attend and contribute to the validation of the data collected from HSCPs. A summary document of the thematic analysis of the family carer and older people's data was sent to each of the non-HSCP interviewees and follow-up phone calls or home visits were made to discuss the tentative findings and gather their feedback. Upon completion of the respondent validation process, a broader research team meeting was held in which participant feedback was discussed and integrated into the inductive thematic structure (DOD, CD, LC, ENS, SD, TK). 
In the final stage of the analysis, the themes identified through inductive coding were transposed onto the conceptual framework described in the rapid realist review by Davies et al (2019). This framework underpins a programme theory categorising mechanisms to support ADM practice according to four domains. The four domains of the programme theory provided the initial macro-thematic structure which was modified and adapted according to the experiences of the study participants as they reflected upon their lived experience of acute care of older people. The result of this analysis is an adapted programme theory for ADM implementation which is inclusive of the lived experiences of the participants and which is responsive to the contextual complexities of decision-making with older people in acute care settings.

Data counts have been illustrated with words such as 'a majority' or 'several' in keeping with the narrative style of the results. The following identifiers are used to present the findings: FC (family carer), OP (older person), OPD (older person with dementia), MD (medical doctor), Ns (nurse), SW (social worker), SLT (speech and language therapist), OT (occupational therapist), Dt (Dietitian), PT (physiotherapist).

\section{Results}

\subsection{Participants}

Tables 1-4 provide a breakdown of the participant $(\mathrm{N}=39)$ characteristics. A total of 26 health and social care professionals working in the acute care of the older person were interviewed. This included 12 allied healthcare professionals consisting of: social workers $(n=4)$, occupational therapists $(n=2)$, dietitians $(n=$ $2)$, speech and language therapists $(n=3)$ and a physiotherapist $(n=1)$. All of the HSCPs had greater than 5 years' experience working in acute care and 15 had greater than 10 years. Interviews ranged from 18 minutes to one hour 10 minutes.

A total of 13 participants were older people $(n=4)$, older people with a diagnosis of dementia $(n=4)$ and family carers $(n=5)$. All of those with a diagnosis of dementia had a recent diagnosis (less than five years). The reason for hospital admission among the older people included stroke, abdominal pain, cancer and ear infection. The family carers were caring for partners $(n=1)$, siblings $(n=2)$ and parents $(n=2)$. The reason for care ranged from Parkinson's disease $(n=1)$; dementia $(n=1)$; cancer $(n=1)$ and intellectual disability $(n=2)$. Interviews ranged from 30 minutes to one hour 50 minutes.

\section{Table 1: Overview of Participant characteristics $(\mathrm{N}=39)$}




\begin{tabular}{|c|c|c|c|c|}
\hline Group & Characteristic & Inclusion criteria & Male & Female \\
\hline \multirow{3}{*}{$\begin{array}{l}\text { Hospital } \\
\text { A } \\
n=11\end{array}$} & Doctors $(n=5)$ & \multirow{6}{*}{$\begin{array}{l}\text { Working in acute care in care for } \\
\text { older person service }\end{array}$} & 1 & 4 \\
\hline & Nurses $(n=0)$ & & & \\
\hline & Allied Health $(n=6)$ & & 1 & 5 \\
\hline \multirow{3}{*}{$\begin{array}{l}\text { Hospital } \\
\text { B } \\
n=15\end{array}$} & Doctors $(n=5)$ & & 0 & 5 \\
\hline & Nurses $(n=4)$ & & 1 & 2 \\
\hline & Allied Health $(n=7)$ & & 0 & 7 \\
\hline \multirow{2}{*}{$\begin{array}{l}\text { Older } \\
\text { people } \\
n=8\end{array}$} & Older People $(n=4)$ & \multirow{2}{*}{$\begin{array}{l}\text { Older person with experience of an } \\
\text { acute care admission in the } \\
\text { previous six months. }\end{array}$} & 4 & 0 \\
\hline & $\begin{array}{l}\text { People with a } \\
\text { diagnosis of } \\
\text { dementia }(n=4)\end{array}$ & & 2 & 2 \\
\hline $\begin{array}{l}\text { Family } \\
\text { Carers } \\
n=5\end{array}$ & $\begin{array}{l}\text { Family Carers } \\
(n=5)\end{array}$ & $\begin{array}{l}\text { Family carer with experience of } \\
\text { accompanying an older person to } \\
\text { acute care setting. }\end{array}$ & 0 & 5 \\
\hline $\begin{array}{l}\text { Total } \\
n=39\end{array}$ & & & 9 & 30 \\
\hline
\end{tabular}

Table 2: Participant Characteristics for Family carers $(\mathrm{N}=5)$

\begin{tabular}{|lllll|}
\hline ID & $\begin{array}{l}\text { Age of } \\
\text { carer }\end{array}$ & $\begin{array}{l}\text { Years in a caring } \\
\text { role }\end{array}$ & $\begin{array}{l}\text { Diagnosis of the } \\
\text { recipient }\end{array}$ & $\begin{array}{l}\text { Relationship with the } \\
\text { recipient }\end{array}$ \\
\hline FC1 & 67 & 11 & Neuromuscular disorder & Wife \\
\hline FC2 & 64 & 23 & Learning disability & Older sister \\
\hline FC3 & 38 & 4 & Dementia & Daughter \\
\hline FC4 & 62 & 35 & Autism & Older sister \\
\hline FC5 & 33 & 2.5 & Cancer & Daughter \\
\hline
\end{tabular}

Table 3: Participant Characteristics for People with a diagnosis of dementia 


\begin{tabular}{|lll|}
\hline ID & Age & Diagnosis \\
\hline OPD1 & 69 & Alzheimer's disease \\
\hline OPD2 & 68 & Alzheimer's disease \\
\hline OPD3 & 55 & Lewy Body Dementia \\
\hline OPD4 & 74 & Alzheimer's disease \\
\hline
\end{tabular}

Table 4: Participant Characteristics for Older People

\begin{tabular}{|lll|}
\hline ID & Age & Reason for admission \\
\hline OP1 & 80 & Melanoma \\
\hline OP2 & 76 & Stroke \\
\hline OP3 & 74 & Gallstone sludge \\
\hline OP4 & 72 & Ear infection \\
\hline
\end{tabular}

\subsection{Personalisation of Health and Social Care}

Personalisation refers to the appreciation of the individuality of the older person within the context of their life experience, personal values, social determinants and their preferences. When the participants were asked to share their experiences of ADM in acute care they consistently framed their narratives using the language of personalisation. The older people and family carers frequently linked decisionmaking with placing the patient at the centre of care. This included reference to speaking directly to the patient (FC2), seeing the person not the diagnosis (OPD3) or recognising the patient as being at the centre of all care decisions (OP2). The HSCP participants described the complex interpersonal work of building therapeutic relationships with their patients. They noted the necessity to take the time to respond to the individual needs of the patients (SLT1). They spoke about the importance of 'individualising' the care they deliver and not using a 'tick box' approach (Ns3). They noted the drive 'to turn over patients quickly' and how that was not conducive to decision-making (MD6). A common theme in these interviews was the juxtaposition of standardised health service delivery, which is task and diagnosis oriented, with a more flexible and adaptive healthcare which is person-centred.

Our analysis has identified 'personalisation' as the central concept in the implementation of assisted decision making in acute care. The programme theory described by Davies et al. (2019), which was used to structure the analysis of the interviews for this article, indicated that several facilitators or inhibitors in three key domains can affect the success of ADM implementation. In the following, we will discuss these 
domains. The reader will note that we have split the 'environmental and social restructuring' domain into two separate domains. (see figure 2).

\subsection{Environment and Resources}

Supporting ADM with older people within acute care settings requires consideration of, and investment in, the physical and structural acute care environment. This encompasses access to resources to support communication and to meet care needs in the community. Older people particularly were frightened and confused by the lack of transparency concerning when and from whom they would receive information or support, the limited privacy for critical conversations and the frantic and seemingly disorderly pace of the acute care environment. Similarly, HSCPs acknowledged that they worked in noisy, high paced environments, mostly focused on the immediate clinical tasks, not understanding or seeing an integrated care pathway involving community providers. The conditions under which they worked made it challenging to communicate effectively. Despite these challenges many reported successful examples of ADM. (See Figure 3)

\section{Older People and Family Carers}

\section{A Disorientating and Disempowering Environment}

Most of the older people, as well as family carers, described chaotic and frightening encounters with the emergency department which was depicted as a "war zone" (OPD3) or a "battlefield" (OP2) where "you are not a priority" (OPD3). A common feature of their accounts was the time spent in acute settings waiting for assessments or procedures and a feeling that they were forgotten by staff who "are all running and racing and busy" (OPD2).

The participants noted the lack of privacy which inhibited conversations; "everything was spoken of in a public area" (OPD1). They described the physical layout of hospitals as being disorienting. They were unsure how to navigate the space and this not only exacerbated symptoms of delirium but also increased their anxiety. This was compounded by multiple ward moves during an admission:

I was moved five times in eleven days. [...] I cannot tell you how frightening that is. [...] So I'm disoriented. I'm not sure where I am. [...] It was just awful. (OPD2)

A disorganised system for sharing information between patients and staff which frequently relied on verbal exchanges in inappropriate physical spaces was felt to be disempowering by the participants. They spoke about the necessity for burdensome repetition:

[...] do I have to go through all this again? [...] I used to be worn out with it, you know[...] look it up in the chart, look it up in the notes, you know, but they couldn't do that. They had to get it every time. I hated that. (FC1) 
They also described not knowing when their team would come on rounds and having to repeatedly request meetings, in one case a participant spoke about following a consultant through the hospital to have a conversation. This was contrasted by positive experiences of strong therapeutic relationships and person-centred conversations with healthcare staff:

He spent probably two hours with me the first day on my own and he probably spent an hour with $\mathrm{H}$. and myself the following day and he kept asking is there any questions you want to know. (OPD3)

\section{Health and Social Care Professionals}

\section{Time, an Integrated Care Pathway and Access to Community Services}

HSCP participants noted that high clinical care demands and patient turnover rates may prevent them from meeting the needs of some older patients for time and support to make assisted decisions: "these conversations they are never cut and dry they are never quick." (Ns3). The participants revealed many examples of successful assisted decision-making with older patients and a common feature of these scenarios was the length of time the patient remained in acute care. Patients were given time to regain capacity and insight into their situations, needs and risks through repeated conversations, information sharing and personalised communication: "so be it if she is six months in the hospital at least she is making that call."(SW2)

The participants noted that the lack of an integrated care pathway prevented them from sharing information as well as accessing services in the community which would assist decision-making with older people. These barriers were evidenced in the interviews as repeated assessment and admission, limitations in accessing patient histories and a disparity between patients' wants and community resources:

... we all want the same thing but the services are not available and therefore somebody cannot go home. So there's a huge gap between what people want and what they can have.(MD6)

Some participants indicated that older people may arrive at acute care after a crisis concerning inadequate community services: "when he first came in he was going home with a home care package because he couldn't get it he went into a nursing home" (SW4). Inadequate community care provision becomes the context for decision-making with older people, for discharge planning and interactions with family members:

...sometimes the family members [...] they're worn out and they're stressed and they're just like 'oh my god we can't take them back' (Ns2)

Maximising older person's decision making and communication competence was made difficult due to overcrowding, noisy, cramped conditions often with only a curtain rail for privacy on a multiple occupancy ward. Support from experienced colleagues was identified as vital in assessing capacity and facilitating communication competence. 
Supporting ADM with older people within acute care settings requires consideration of and investment in the physical and structural acute environment. This encompasses the pace of acute care, the requirement for time as well as access to resources to support communication and resources to meet social and care needs in the community. Furthermore, the physical and sensory environment in which acute care is delivered was commonly cited as being of importance for personalisation and ADM practice with older people.

\subsection{Social Restructuring}

Acute care is delivered by multiple professions in the context of multi-disciplinary teams (MDT). Social restructuring refers to strengthening the communication and collaboration among clinical care providers from different disciplines. This involves, for example, interactions grounded in mutual respect, distributed leadership styles and the central involvement of older persons and their family carers or decisionsupporters. The latter, in particular, requires delicate balancing between duty of care on the part of the HSCPs and needs and preferences of patients and families. (see Figure 4)

\section{Older People and Family Carers}

\section{The Inclusive Team}

The older people and family carers described HSCPs talking directly to them and providing them with information in such a way as to facilitate their comprehension: "make it simple for me" (FC1). This assisted them to develop insight into their situation.

Feeling valued and respected within care decision-making was identifiable in the data both from its absence but also from its presence. For example, an older person with a diagnosis of dementia spoke about expressing a contrasting viewpoint to a consultant in a decision around medication rationalisation:

He said you are right we will do it that way we will drop it in fives [...] Right there and then I knew I could trust him I knew I could talk to him (OPD3)

Being valued as a member of an inclusive care team was a strong theme in the family carer accounts. They posited that the knowledge and insights they have about the care needs of the individual (FC2) should make them an equally valuable care team member as the nurse or doctor. They saw themselves as a patient advocate, the person's "voice" (FC3) and the key to enabling a person's will and preference:

...they weren't my team they were my mother's team. But they enabled me to make sure that their patient's wishes came true because they supported me as much as they supported her (FC5)

\section{Health and Social Care Professionals}

Patient Advocacy and Interprofessional Working 
The HSCPs described the challenges of advocating for patients in a complex social context and ensuring that the older person's voice is represented in decision-making conversations. They spoke about the challenge of negotiating different perspectives particularly in situations of family dysfunction:

...certain thresholds were never going to be met because the family hadn't displayed a level to put those in place [...] At the end of the meeting it was clear that they weren't going to be able to meet what the community felt was required for a safe discharge home. (SW3)

The participants spoke of the conflict they experienced in balancing the needs and wishes of the family and their older patients and the difficulty they had in separating these two social and relational aspects of older people's care:

It's hard not to listen to that person because [...] maybe they are completely exhausted they have their own personal life they are working full time. (Ns4)

The necessity to balance the diverging wishes of family and patients created a pressurised environment for the HSCP participants who were advocating for a patient's decision-making in the absence of community care supports to realise those decisions. This pressure was noted as influencing an assessment of decision-making capacity:

If someone is sitting in-between do they or do they not have capacity. I think when family weigh in on top of that and the consultant can sometimes lean towards the patient not having capacity (OT2)

The pressure coming from an under-resourced context for decision-making was also noted as influencing the inter-personal interactions between the HSCPs and their patients whereby they had to manage the expectations of their patients towards more practical or realisable goals. In its most negative form this manifested as the patient being persuaded into a decision which was not aligned to their preference, but which was practically realisable:

A patient whose being, whose arm is being twisted basically and who was traditionally told all sorts of rubbish about you know they'll be going for... 'we'll go and try it for maybe a week or two of convalescence and see how it goes', knowing that that was the plan going forward. (MD6)

The participants commonly aligned highly cohesive inter-professional teamwork with successful and effective ADM. This was particularly the case among teams which fostered trust through sharing and valuing of disciplinary expertise and diagnostic information as well as collective decision-making:

Each member of the multidisciplinary team brings a different skill and a different perspective and each has their own area of expertise [...] I think the consultant is a member of the multidisciplinary team and I think that the team help inform the decision of the consultant (MD6)

While acknowledging the hierarchical consultant-led MDT model, the participants widely agreed that nursing and allied health therapists are best positioned to build a therapeutic relationship by the amount 
of time spent directly with the patient. The skillset of Occupational and Speech and Language Therapists were widely recognised as critical to effective ADM particularly with assessing functional capacity as well as maximising communication competence.

ADM and personalisation in the acute care of older people requires a re-structuring of the social environment in which care is negotiated, managed, planned and delivered. This implementation domain challenges acute care settings to develop inclusive care teams which promote respectful interprofessional collaboration, distributed leadership styles and involvement of older patients and their family carers or decision-supporters.

\subsection{Education, Training and Enablement}

Fostering a strong learning culture which will enable reflective practice and confidence about the processes of ADM among HSCPs is critical to the implementation of ADM in the acute care of older people. One of the biggest challenges for HSCPs, family carers and patients is the fluctuating capacity to process and act on information among people with impaired cognitive functioning, especially those with dementia. HSCPs not only require a comprehensive understanding of the clinical and social circumstances that are associated with different degrees of alertness, awareness and cognitive capacity, but also the requisite skills to respond, interact and engage with their patients over the course of their illness. This necessitates continuing professional education and upskilling. (See Figure 5)

\section{Older People and Family Carers}

\section{Capacity, Cognition and Communication}

These participants connected training in dementia to person-centred care through understanding how to communicate with, and holistically treat, an older patient with dementia. One of the participants explained how her anxiety, associated with her dementia, was mismanaged during her acute admission because of a lack of understanding: "Every time I was moved it brought out more anxiety within me that I had to cope with." (OPD2) A family carer recounted accompanying her mother who had dementia and who was suspected to be experiencing a stroke to the hospital. The carer explained how the HSCPs lack of knowledge of dementia led to difficulty in communicating with and assessing her mother:

...like lift the arms and yeah and she wasn't able to do them, not because it was a stroke but because she couldn't understand what they were trying to get her to do.[...] speak slower and maybe to try to guide her of what to do [...]not how you would deal with a person with dementia. (FC3)

They also discussed HSCPs understanding of ADM with patients who have fluctuating capacity. They describe HSCPs who effectively manage this as understanding the distinction between capacity and cognition and how to support patient's will and preference through discerning use of decision supporters.

\section{Health and Social Care Professionals}




\section{Training, Reflection and Communication}

The fluctuating and individualised nature of capacity were reported by the HSCP participants as being frequently misunderstood in the acute care setting. They provided many examples of the time-consuming and iterative process of supporting the decision-making of patients who have cognitive or communication impairments. They described an overreliance on assessments of cognition as indicative of decision-making capacity. They noted that there was often confusion among professionals regarding the distinction between cognition and capacity and this was particularly complicated for patients with a communication disorder (SLT3).

HSCP participants gave detailed accounts of their decision-making processes, they spoke of "really agonising over it" (MD5), "measuring the balance" between risk and patient's will (SW1) and assessing whether the "right decision is being made" (SW2). This need for reflection on decision making was a common feature across their accounts. Advance Care Planning (ACP) was frequently referenced as a potential enabler for HSCPs in understanding the will and preferences of patients. However, they acknowledged the lack of awareness among the general public regarding ACP: "some people haven't a clue they haven't heard of it." (MD6). They identified skills required by HSCPs to engage in conversations around ACP:

...it is a horrible conversation to have with somebody but it's a practical one you know. [...]there is no cure you know and future planning is the only way that you can take control back (Ns4)

Inter-personal communication skills were shown to be essential to person-centred care of older people and delivering a collaborative approach to care planning including the patient, their family as well as the inter-professional team:

[the consultant] took my decision on board and said well look if that is what you feel the patient needs we will go with that collectively. We will give it a shot at home again and we will look at long term care for the next option so the patient was happy and the family were happy with that (SW2)

Building capacity was inextricably linked to the personalisation of care by the participants as they spoke about maximising patient insight through effective communication for an informed decision. They described "simplify(ing) the language" (NS3); "we've always had to kind of adapt the way we ask questions" (SLT1); using communication aids: "providing the teams with books of photos, pictures, imagery, strategies to use, for the rest of the team to use in communication with the patient" (MD6); paying attention to non-verbal cues: "reading what they look like during therapy." (PT1) and repeating information to allow for its absorption by the patient:

it's a slow it's repeated discussion after discussion very much the same content but it's just bringing people to that slow realisation (Ns3).

They also individualised their approach to patients to allow for fluctuating capacity: "always saw him at a very good time of day for him" ( MD6). Of utmost importance, however, was their recognition of the 
necessity for time and a focus on the goals and wishes of their patients.

Fostering a strong learning culture which will enable reflective practice and confidence in relation to the processes of ADM among HSCPs is critical to implementation of ADM in the acute care of older people. This pertains to both formal and informal educational mechanisms for HSCPs to acquire knowledge, skills and confidence in their own processes for maximising patient capacity and assisting their decisionmaking. This implementation domain also encompasses the necessity for public enablement through care planning in relation to their will and preferences and their personal values.

\subsection{Culture and Leadership}

Culture and leadership provide the broader social context for ADM. Both can be 'fertile ground' for growing positive ADM practices. Conversely, a cultural context reluctant to change; adhering to outdated command and control practices and embracing hierarchical, centralised leadership structures can seriously inhibit the adoption of ADM. The implementation domains of education and training converge with the environmental and social context for ADM to influence the individual and organisational behaviours within a culture of acute care for older people. This relates to the value system underpinning the acute care setting and how those values are expressed through power relations, language, communication and prioritisation of metrics and resources. For older people and family carers, being seen, valued and acknowledged as individuals is a critical feature of a positive ADM culture. From the perspective of HSCPs, what matters most is being trusted and encouraged to make decisions, and not to be held back by a context that is fundamentally risk averse. (See Figure 6)

\section{Older People and Family Carers}

\section{Depersonalisation versus Visibility}

These participants provided a strong sense of being lost within a culture of care which at times made them feel invisible. They waited for procedures as inpatients without information as to when they would be seen. They spoke about feeling afraid to fall asleep in case they missed their care team's rounds and the opportunity to speak to their consultant. They articulated a sense of being a cultural outsider, lost in the codifying language of the acute care setting such as different coloured staff uniforms (OP3). They noted a pace of acute care which was overwhelming and they described multiple ward moves in one admission leading to disorientation. One older person described a particularly traumatic experience of his catheter bag overflowing, despite frequent requests to nursing staff to change the bag: "I said it to them on two occasions you know that it's not a nice sensation at night...that feeling you know"(OP3). All of these examples conveyed a value system which disempowered the individual in favour of organisational expediency or rationalisation. As one older person with dementia exclaimed 'it was easier to sob into my pillow than to just go and ask again' (OPD2). 
The requirement for leadership in developing a culture of personalisation through individual behaviour was conveyed through a family carer's account of feeling like she 'mattered' within the organisation of her mother's care:

He realised this is a family losing somebody and it means something it means something to them. [...] what mattered was answering our questions what mattered was being available when we needed him to be available. (FC5)

This account of feeling visible contrasts with the other experiences of depersonalisation and demonstrates very effectively the power of leaders in expressing the values of personalisation through their interpersonal interactions.

\section{Health and Social Care Professionals}

\section{Interventionism, Risk Tolerance and Power Dynamics}

The HSCPs described the challenge of managing the complex care needs of older people in a fast-paced, interventionist acute culture. The participants spoke about working against time pressure to personalise patient care, maximise insight and advocate for patients' wishes: "we're under such pressure here that it's hard to think of, to remember the patient's kind of wishes."(SLT1)

The participants noted that the acute setting was characterised by a culture of risk aversion which when coupled with a lack of advance care planning rendered the expression of a person's will and preference in decision-making very challenging. Some participants spoke about the need for a shift towards a more risk-tolerant culture which would provide patients with the opportunity to succeed in positive outcomes for some potentially risky decisions: "she was given the, I guess the opportunity to fail on discharge, she was ultimately discharged home"(MD6). There was, however, also a sense of unease among some participants who argued that perhaps the legislation placed too much emphasis on developing a culture of risk tolerance which would lead to potentially harmful outcomes for patients:

We have all worked on best interest for years. [...] I do have a slight fear that we may be so heavily on will and preference that we almost throw the baby out with the bathwater (MD9)

The participants spoke in great detail about the challenge of weighing a patient's will and preferences against their insight into the risks associated with a decision. This was particularly challenging in a culture of risk aversion and where there were conflicting opinions within healthcare teams and from family regarding the patient's capacity. They spoke about the process as 'agonising' (MD5) and involving 'difficult conversations' within teams and with patients or family:

If you know that there is a family member who I would say would take litigation like at the first drop of something going wrong so it had to be really [...] that was quite you know intimidating (SW1) 
In advocating for their patient's decision-making capacity they often found themselves and at the centre of conflicting power dynamics played out in a culture of risk aversion and hierarchical physician-led decision-making. The effect of this top-down cultural context was to create differential access to power and therefore restrain individual influence within a decision-making process. In its extreme form this topdown culture muted the influence and voice of the older person.

The implementation domain of culture and leadership describes the organisational commitment to a shared vision for ADM and personalisation within the acute care of older people. This relates to the value system underpinning the acute care setting and how those values are expressed through power relations, language, communication and prioritisation of metrics and resources.

\section{Discussion}

Personalisation of Healthcare was identified as a core variable of ADM which enables genuine choice and control for older people on matters related to their care. Taking the current practices and dynamic contextual complexities into account, the PT identified four key areas for attention and investment. These are (1) Environment and Resources, (2) Social Restructuring (3) Education, Training \& Enablement and (4) Culture and Leadership.

Older people, family carers and HSCPs alike described several weaknesses in the acute care system for quality ADM practice. The participants' accounts of the environment in which care was managed, highlighted the inappropriateness of the physical and social infrastructure in acute settings for decisionmaking with older people, particularly those experiencing cognitive impairment. There were examples of HSCPs personalising their interactions to build their patients' decision-making capacity. However, these examples of individualised relational work were undertaken in an acute environment which presented systematic obstacles relating to accessing resources, inappropriate physical infrastructure and pressure to discharge patients. Individualised relational work of HSCPs has been previously reported as a mediator of powerful institutional drivers that may otherwise result in negative experiences and poor care (Bridges et al., 2020).

The participants noted that managing fluctuating capacity or episodes of acute delirium may be a feature of complex care for older people. They noted that these patients might require time and flexibility in the system to maximise insight and ascertain preferences. As Donnelly (2019) suggests, key within care for patients with dementia is a value for the personhood of the patient. Ascertaining and respecting their current will and preference is time-consuming and complex, as our participants consistently noted. Accomplishing this requires a restructuring of the physical and social environment in which care is negotiated, managed, planned and delivered (Bridges et al., 2020) The chaotic acute care environment was consistently identified as incompatible with the care of older people with dementia and those with fluctuating capacity. The reduction of standardised approaches to care and the revision of metrics to measure practices for building capacity and recognising the individual or team efforts for personalising care could build a more responsive healthcare system (Bridges et al., 2019; Ní Shé et al., 2020). 
Alongside reform of the physical and social systems of care, education and training are required to provide all HSCPs with the knowledge and skills to undertake the complex work of building decisionmaking particularly in the context of fluctuating capacity (Bradshaw, 2000; Dukes and McGuire, 2009) Donnelly (2019) notes that this is particularly important in addressing the tension between protectionism and a human rights approach to decision-making. People with dementia are at a greater risk of abuse than people with other forms of disability, which adds complexity to ADM practice (Donnelly, 2019). The risk of elder abuse means that practitioners must balance their duty of care towards vulnerable patients against their right to autonomous decision-making.

Strong interpersonal communication skills frequently emerged from the participants' accounts as a critical component of the education, training and competence of all HSCPs. Building capacity may require a 'total communication approach' where HSCPs utilise communication aids and augmentative and alternative communication (Bradshaw, 2000) For effective individualised communication to support ADM in acute care settings, specific guidelines will be required. Participant experience emphasised the need to support implementation through the development of a strong learning culture that enables reflective practice and develops confidence in ADM among HSCPs.

This study identified that education/training and the environmental and social context for ADM influence the individual and organisational behaviours within a culture of acute care for older people. The participants described a culture of risk aversion which emphasises documentation and audit, discourages independent decision-making, and elevates safety at all costs over choice and individual agency. It was argued that systems of documentation would need to be changed within the culture of care to facilitate recording of HSCP decision-making and their efforts in building their patient's decisionmaking capacity. Furthermore, opportunities for inter-professional reflection, mentoring and guidance would assist HSCPs to develop their competencies as well as their confidence regarding ADM with older patients, particularly those with cognitive impairment or who may be at risk of harm (Levinson, 2011)

The HSCP participants described an acute culture which is characterised by an emphasis on intervention and patient turnover within a context of risk aversion, hierarchical decision-making and lack of integration for patients transferring across services. This had a disempowering effect not only on older people but in some instances on the HSCP themselves. They were challenged to advocate for their patient's capacity for decision-making within a context of risk management and differential access to power in the decisionmaking process. They often positioned themselves as advocates for their patients. This was particularly evident where they felt that a patient could become disempowered within a system of care which is under-resourced to facilitate decision-making as well as pressurised in terms of conflicting wishes and needs of various stakeholders. ADM was most successful when there was collective decision-making among patients, family and an MDT and where the patient's wishes and preferences carried the strongest weight. This involved strong interprofessional collaboration and trust as well as value for all stakeholder input. 


\subsection{Study Contribution}

This study demonstrates the importance of qualitative methodology for validation of realist programme theories ensuring they are grounded in lived experience and responsive to relevant sociological and contextual factors. Furthermore, our study illuminates the contribution qualitative research can make to broader salient debates on healthcare context, implementation and responsiveness (May et al., 2016; Ní Shé et al., 2020; Pfadenhauer et al., 2017).

As the health system in Ireland prepares to implement ADM legislation, this study provides timely, salient information on the socio-cultural influences that might enable or hinder its implementation for older people in hospital settings. The adapted programme theory resulting from this study can inform implementation planning and is relevant for government policymakers, healthcare organisations, educators, researchers and patient associations. Within the international arena, this theory can be applied to other healthcare settings undergoing similar legislative changes in line with the UNCRPD. While it is not possible to draw generalisable conclusions, this study provides methodological guidance and a coding framework for a similar pre-implementation evaluation to explore stakeholder's experiences and perceptions.

\subsection{Limitations and further research}

The data represent participants' subjective accounts of their experiences of ADM within two acute care sites. While it was a comprehensive analysis, it does not include a full systematic conceptualisation and assessment of context and ADM implementation. Data were collected as part of a cross-sectional and not prospective study. Accounts may be prone to recall and social desirability bias. Further, prospective studies may consider appropriate objective measures, case note reviews or ethnographic observations of ADM in practice over time. Furthermore, recruitment was limited to two hospitals and patient advocacy groups. The inclusion of other health care sites and respondent groups would have increased diversity. We encourage other researchers to apply, test and further refine the programme theory presented in this article.

\section{Conclusion}

The study highlights the importance of understanding socio-cultural and political contexts when trying to practically support implementation planning for ADM. Realist reviews and syntheses have gained popularity in the context of healthcare-related implementation research. Through the application of a qualitative analysis of the decision-making experiences of key stakeholders in acute care settings, this article adapted a programme theory for the implementation of ADM policy. The article illuminates the mechanisms that facilitate or inhibit the implementation of healthcare innovations and policies, with a particular focus on ADM implementation with older people in acute care. The adapted programme theory which resulted from the qualitative exploration of decision-making practice informs policy and practice 
planning in Ireland. It also contributes to wider debates internationally on the role of socio-cultural and political contexts for ADM implementation.

\section{List Of Abbreviations}

ADM Assisted Decision-Making

UNCRPD UN Convention on the Rights of Persons with Disabilities

HSCPs Health and Social Care Professionals

RRR Rapid Realist Review

FC Family Carer

OP Older Person

OPD Older Person with Dementia

MD Medical Doctor

Ns Nurse

SW Social Worker

SLT Speech and Language Therapist

OT Occupational Therapist

Dt Dietician

PT Physiotherapist

MDT Multi-Disciplinary Team

ACP Advance Care Planning

\section{Declarations}

\subsection{Ethics and Integrity Statement}

The study was approved by the University College Dublin Human research ethics committee in 2018 (REC reference LS-18-73-ODonnell). All research methods including participant recruitment and data collection, were carried out in accordance with relevant guidelines and regulations. Informed consent for participation in the study was obtained from all subjects. The production of written communication material followed the National Adult Literacy Guidelines (NALA) for accessible communication. The management of research data has been in line with requirements set out under the European Union's general data protection regulation and Irish data protection legislation.

Each of the authors confirms that this manuscript has not been previously published and is not currently under consideration by any other journal. Additionally, all of the authors have approved the contents of this paper and have agreed to the submission policies of BMC Health Services Research.

\subsection{Consent for Publication}


Informed consent for publication of anonymised and de-identified data was obtained from all research participants.

\subsection{Availability of Data and Materials}

The anonymised data that support the findings of this study are available from the corresponding author, upon reasonable request.

\subsection{Competing Interests}

The authors declare that there is no conflict of interest.

\subsection{Funding}

This research and publication was supported by the Health Research Board, Ireland [APA-2016-1878].

\subsection{Authors Contributions}

Deirdre O'Donnell: Conceptualization-Equal, Formal analysis-Equal, Investigation-Equal, MethodologyEqual, Project administration-Lead, Writing-review \& editing-lead.

Carmel Davies: Conceptualization-Equal, Formal analysis-Equal, Investigation-Equal, Methodology-Equal, Writing-review \& editing-Supporting

Lauren Christophers: Formal analysis-Equal, Investigation-Supporting, Project administration-Supporting, Writing-review \& editing-Supporting

Eidín Ní Shé: Conceptualization-Equal, Investigation-Equal, Methodology-Equal, Writing-review \& editingSupporting

Sarah Donnelly: Conceptualization-Equal, Investigation-Equal, Methodology-Equal, Writing-review \& editing-Supporting

Thilo Kroll: Conceptualization-Equal, Formal analysis-supporting, Funding acquisition-Lead, InvestigationEqual, Methodology-Equal, Writing-review \& editing-supporting

\subsection{Acknowledgement}

The authors wish to acknowledge and thank the participants who agreed to take part in this study and who provided such valuable insights into their experiences. We also wish to thank the members of the PADMACS project advisory groups for their advice and feedback. We would like to thank our public and patient representation for enabling access to their members for interviews in particular Dr Bernadette Rock from The Alzheimer Society of Ireland; Dr Nikki Dunne, Family Carers Ireland and Ms. Bibiana Savin, 
SAGE Advocacy, Ireland. We also acknowledge the contribution of Dr Francesco Fattori to the initial phases of this project and thank him for his contribution to participant recruitment and data collection.

\subsection{Source Permissions}

Figure 1: Four Programme Theory Domains for Promoting ADM in Health and Social Care Practice has been reproduced with authors' permission from Davies, C., Fattori, F., O’Donnell, D., Donnelly, S., Ní Shé, É., O. Shea, M., Prihodova, L., Gleeson, C., Flynn, Á., Rock, B., Grogan, J., O’Brien, M., O'Hanlon, S., Cooney, M. T., Tighe, M., \& Kroll, T. (2019). What are the mechanisms that support healthcare professionals to adopt assisted decision-making practice? A rapid realist review. BMC Health Services Research, 19(1), 960. https://doi.org/10.1186/s12913-019-4802-x

\section{References}

1. Boyle, G., 2011. Early implementation of the Mental Capacity Act 2005 in health and social care. Crit. Soc. Policy 31, 365-387. https://doi.org/10.1177/0261018311398781

2. Bradshaw, J., 2000. A Total Communication Approach Towards Meeting the Communication Needs of People with Learning Disabilities. Tizard Learn. Disabil. Rev. 5, 27-30. https://doi.org/10.1108/13595474200000005

3. Bridges, J., Collins, P., Flatley, M., Hope, J., Young, A., 2020. Older people's experiences in acute care settings: Systematic review and synthesis of qualitative studies. Int. J. Nurs. Stud. 102, 103469. https://doi.org/10.1016/j.ijnurstu.2019.103469

4. Bridges, J., Pope, C., Braithwaite, J., 2019. Making health care responsive to the needs of older people. Age Ageing 48, 785-788. https://doi.org/10.1093/ageing/afz085

5. CSO, 2017. Census 2016 Summary Results - Part 2 Chapter 9 Health, disability and caring. Central Statistics Office, Dublin.

6. Davies, C., Fattori, F., O’Donnell, D., Donnelly, S., Ní Shé, É., O. Shea, M., Prihodova, L., Gleeson, C., Flynn, Á., Rock, B., Grogan, J., O’Brien, M., O’Hanlon, S., Cooney, M.T., Tighe, M., Kroll, T., 2019. What are the mechanisms that support healthcare professionals to adopt assisted decision-making practice? A rapid realist review. BMC Health Serv. Res. 19, 960. https://doi.org/10.1186/s12913-0194802-x

7. Devi, N., 2013. Supported Decision-Making and Personal Autonomy for Persons with Intellectual Disabilities: Article 12 of the UN Convention on the Rights of Persons with Disabilities. J. Law. Med. Ethics 41, 792-806. https://doi.org/10.1111/jlme.12090

8. Devi, N., Bickenbach, J., Stucki, G., 2011. Moving towards substituted or supported decision-making? Article 12 of the Convention on the Rights of Persons with Disabilities. Alter 5, 249-264. https://doi.org/10.1016/j.alter.2011.07.002 
9. Donnelly, M., 2019. Deciding in dementia: The possibilities and limits of supported decision-making. Int. J. Law Psychiatry 66, 101466. https://doi.org/10.1016/j.jilp.2019.101466

10. Dukes, E., McGuire, B.E., 2009. Enhancing capacity to make sexuality-related decisions in people with an intellectual disability. J. Intellect. Disabil. Res. 53, 727-734. https://doi.org/10.1111/j.13652788.2009.01186.x

11. Giampieri, M., 2012. Communication and informed consent in elderly people. MINERVA Anestesiol. $78,7$.

12. Griffith, R., Tengnah, C., 2008. Mental Capacity Act 2005: assessing decision-making capacity 2 . Br. J. Community Nurs. 13, 284-288. https://doi.org/10.12968/bjcn.2008.13.6.29463

13. James, K., Watts, L., 2014. Understanding the Lived Experiences of Supported Decision-Making in Canada.

14. Kelly, B.D., 2017. The Assisted Decision-Making (Capacity) Act 2015: what it is and why it matters. Ir. J. Med. Sci. 1971 - 186, 351-356. https://doi.org/10.1007/s11845-016-1443-5

15. Knight, F., Kokanović, R., Ridge, D., Brophy, L., Hill, N., Johnston-Ataata, K., Herrman, H., 2018. Supported Decision-Making: The Expectations Held by People With Experience of Mental Illness. Qual. Health Res. 28, 1002-1015. https://doi.org/10.1177/1049732318762371

16. Levinson, W., 2011. Patient-centred communication: a sophisticated procedure. BMJ Qual. Saf. 20, 823-825. https://doi.org/10.1136/bmjqs-2011-000323

17. Livingston, G., Leavey, G., Manela, M., Livingston, D., Rait, G., Sampson, E., Bavishi, S., Shahriyarmolki, K., Cooper, C., 2010. Making decisions for people with dementia who lack capacity: qualitative study of family carers in UK. BMJ 341, c4184-c4184. https://doi.org/10.1136/bmj.c4184

18. May, C.R., Johnson, M., Finch, T., 2016. Implementation, context and complexity. Implement. Sci. 11, $1-12$.

19. McCormack, B., McCance, T., Klopper, H., 2017. Person-centred practice in nursing and health care: theory and practice.

20. McDonald, A., 2010. The Impact of the 2005 Mental Capacity Act on Social Workers' Decision Making and Approaches to the Assessment of Risk. Br. J. Soc. Work 40, 1229-1246. https://doi.org/10.1093/bjsw/bcq021

21. McGilton, K.S., Vellani, S., Yeung, L., Chishtie, J., Commisso, E., Ploeg, J., Andrew, M.K., Ayala, A.P., Gray, M., Morgan, D., Chow, A.F., Parrott, E., Stephens, D., Hale, L., Keatings, M., Walker, J., Wodchis, W.P., Dubé, V., McElhaney, J., Puts, M., 2018. Identifying and understanding the health and social care needs of older adults with multiple chronic conditions and their caregivers: a scoping review. BMC Geriatr. 18, 231. https://doi.org/10.1186/s12877-018-0925-x

22. Ní Shé, É., O’Donnell, D., Donnelly, S., Davies, C., Fattori, F., Kroll, T., 2020. "What Bothers Me Most Is the Disparity between the Choices that People Have or Don't Have": A Qualitative Study on the Health Systems Responsiveness to Implementing the Assisted Decision-Making (Capacity) Act in Ireland. Int. J. Environ. Res. Public. Health 17, 3294. https://doi.org/10.3390/ijerph17093294 
23. O’Donnell, D., Ní Shé, É., Davies, C., Donnelly, S., Cooney, T., O’Coimin, D., O’Shea, D., Kyne, L., O'Sullivan, D., Rock, B., O’Shea, M., McAuliffe, E., O'Shaughnessy, A., Kroll, T., 2018. Promoting assisted decision-making in acute care settings for care planning purposes: Study protocol. HRB Open Res. 1, 2. https://doi.org/10.12688/hrbopenres.12797.1

24. Pfadenhauer, L.M., Gerhardus, A., Mozygemba, K., Lysdahl, K.B., Booth, A., Hofmann, B., Wahlster, P., Polus, S., Burns, J., Brereton, L., Rehfuess, E., 2017. Making sense of complexity in context and implementation: the Context and Implementation of Complex Interventions $(\mathrm{CICl})$ framework. Implement. Sci. 12, 21. https://doi.org/10.1186/s13012-017-0552-5

25. Phelan, A., Rickard-Clarke, P., 2020. Person Centred Approaches in Capacity Legislation, in: Phelan, A. (Ed.), Advances in Elder Abuse Research: Practice, Legislation and Policy, International Perspectives on Aging. Springer International Publishing, Cham, pp. 23-37. https://doi.org/10.1007/978-3-03025093-5_3

26. Smith, J., Firth, J., 2011. Qualitative data analysis: the framework approach. Nurse Res. 18, 52-62. https://doi.org/10.7748/nr2011.01.18.2.52.c8284

27. Timmons, S., Manning, E., Barrett, A., Brady, N.M., Browne, V., O'Shea, E., Molloy, D.W., O’Regan, N.A., Trawley, S., Cahill, S., O'Sullivan, K., Woods, N., Meagher, D., Ni Chorcorain, A.M., Linehan, J.G., 2015. Dementia in older people admitted to hospital: a regional multi-hospital observational study of prevalence, associations and case recognition. Age Ageing 44, 993-999. https://doi.org/10.1093/ageing/afv131

28. United Nations General Assembly, 2007. Convention on the Rights of Persons with Disabilities. 29. Yon, Y., Mikton, C.R., Gassoumis, Z.D., Wilber, K.H., 2017. Elder abuse prevalence in community settings: a systematic review and meta-analysis. Lancet Glob. Health 5, e147-e156. https://doi.org/10.1016/S2214-109X(17)30006-2

\section{Figures}




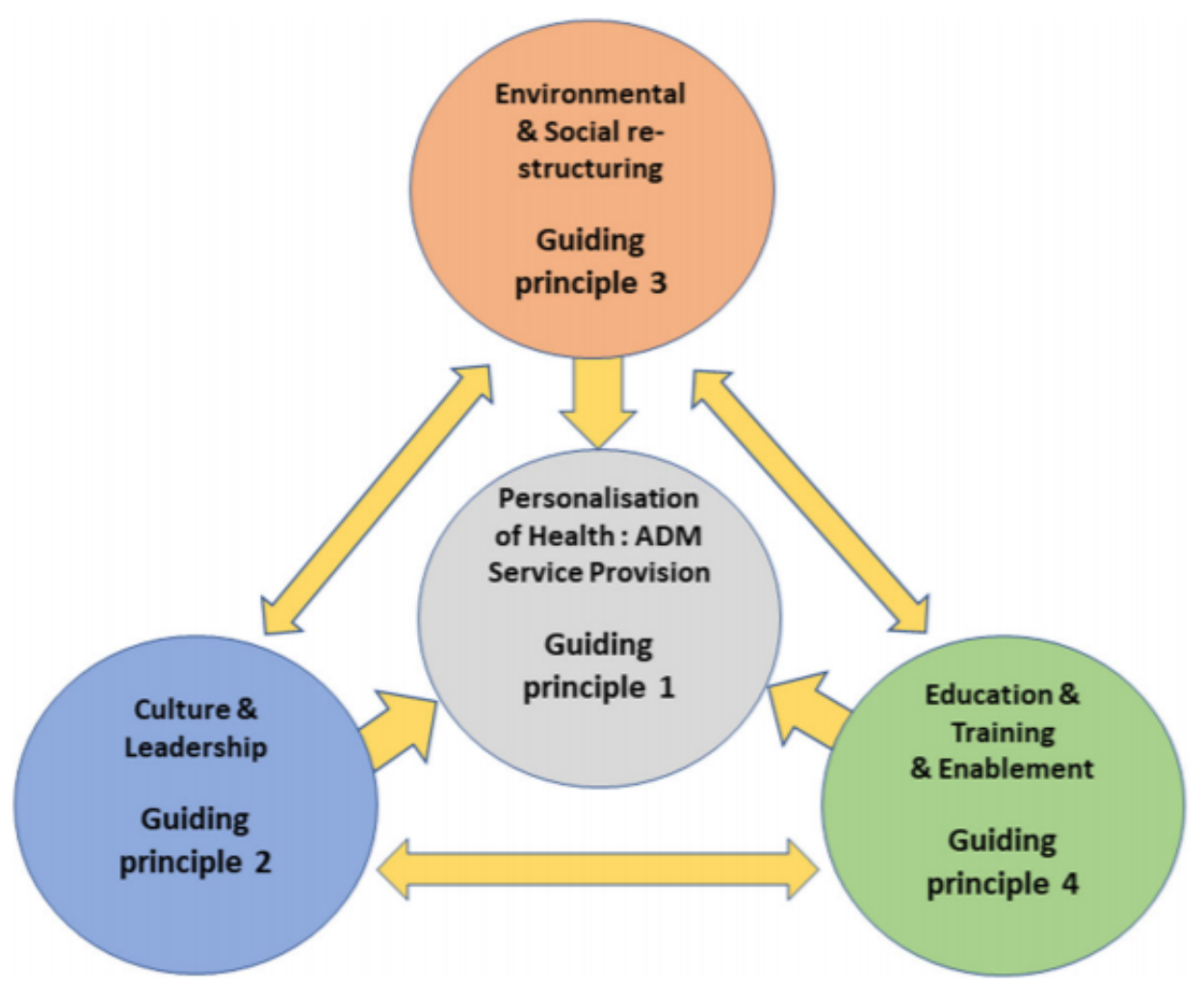

\section{Figure 1}

Four Programme Theory Domains for Promoting ADM in Health and Social Care Practice has been reproduced with authors' permission from Davies, C., Fattori, F., O’Donnell, D., Donnelly, S., Ní Shé, É., O. Shea, M., Prihodova, L., Gleeson, C., Flynn, Á., Rock, B., Grogan, J., O’Brien, M., O’Hanlon, S., Cooney, M. T., Tighe, M., \& Kroll, T. (2019). What are the mechanisms that support healthcare professionals to adopt assisted decision-making practice? A rapid realist review. BMC Health Services Research, 19(1), 960. https://doi.org/10.1186/s12913-019-4802-x 

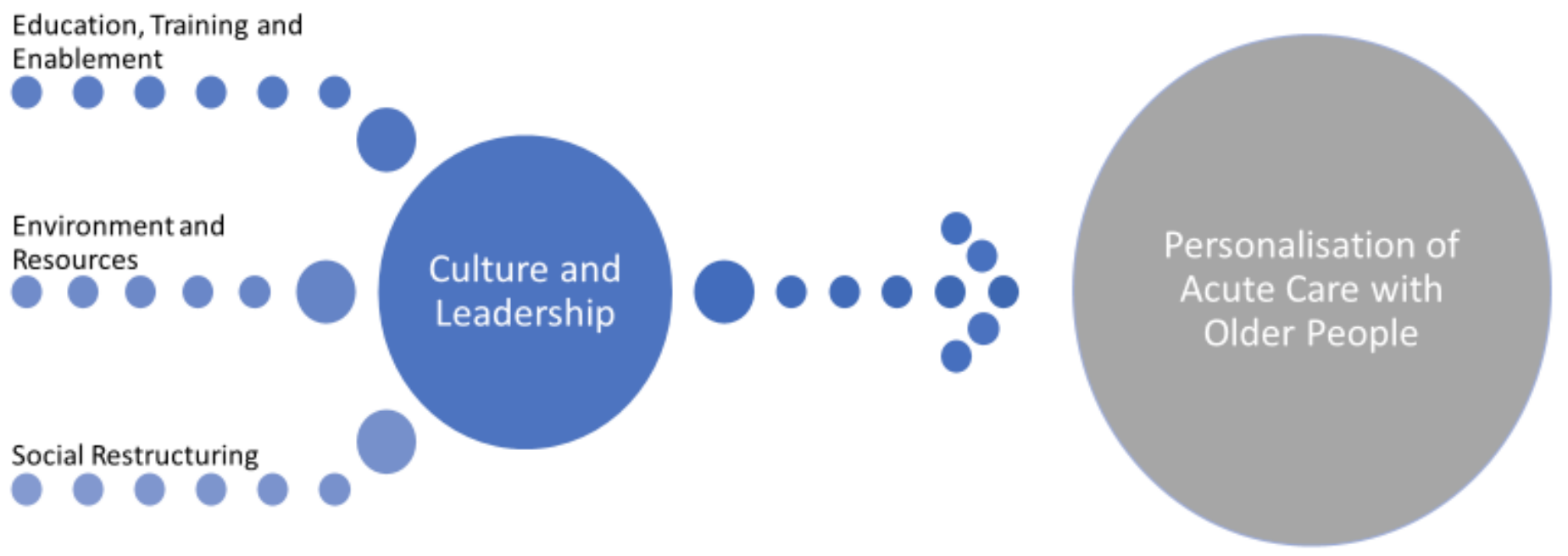

\section{Figure 2}

Implementation Domains for Promoting Personalisation in the Acute Care of Older People

\section{OLDER PEOPLE AND FAMILY CARERS}

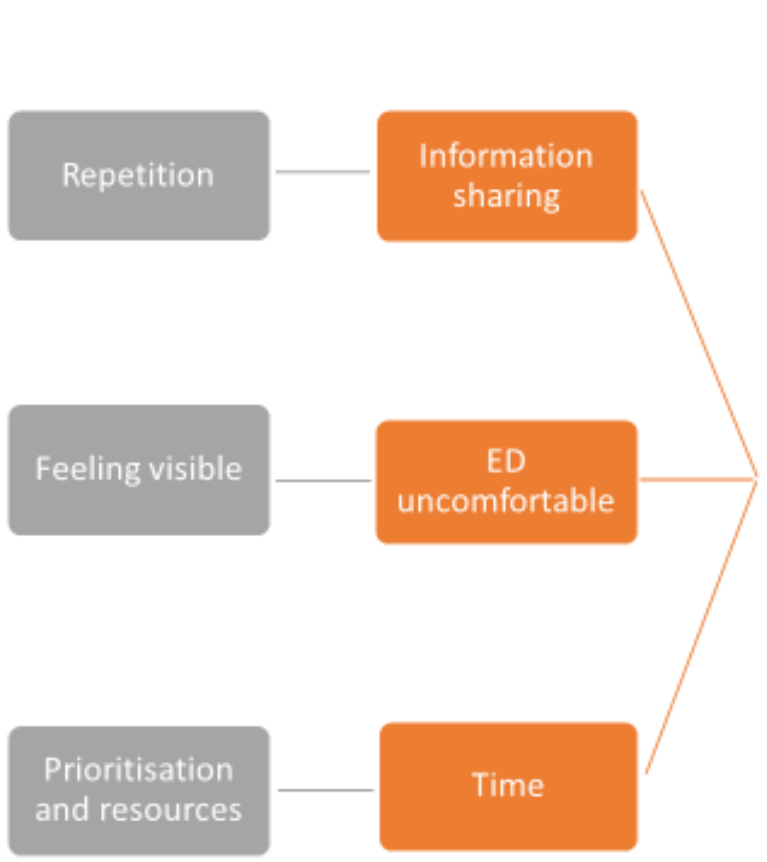

\section{HEALTH AND SOCIAL CARE PROFESSIONALS}

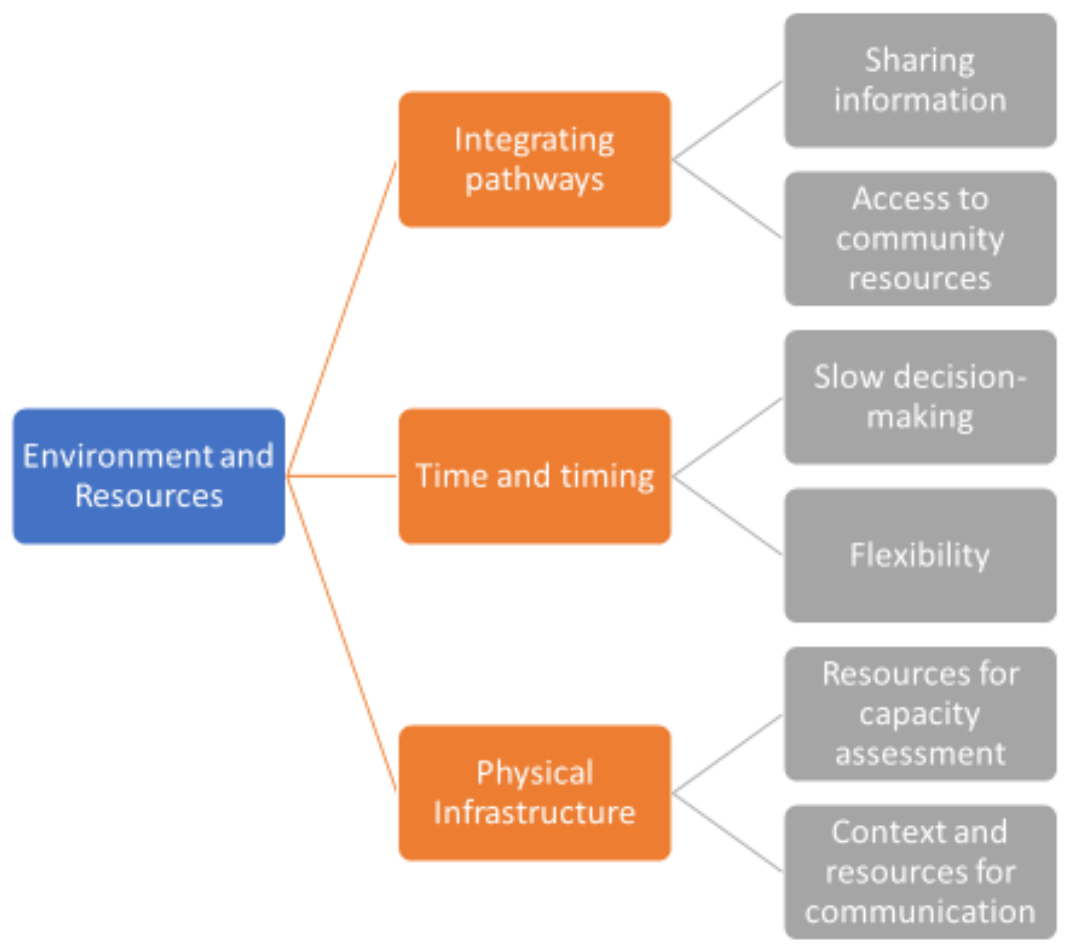




\section{Figure 3}

Overview of the thematic structure of participant data under the domain of environment and resources

OLDER PEOPLE AND FAMILY CARERS

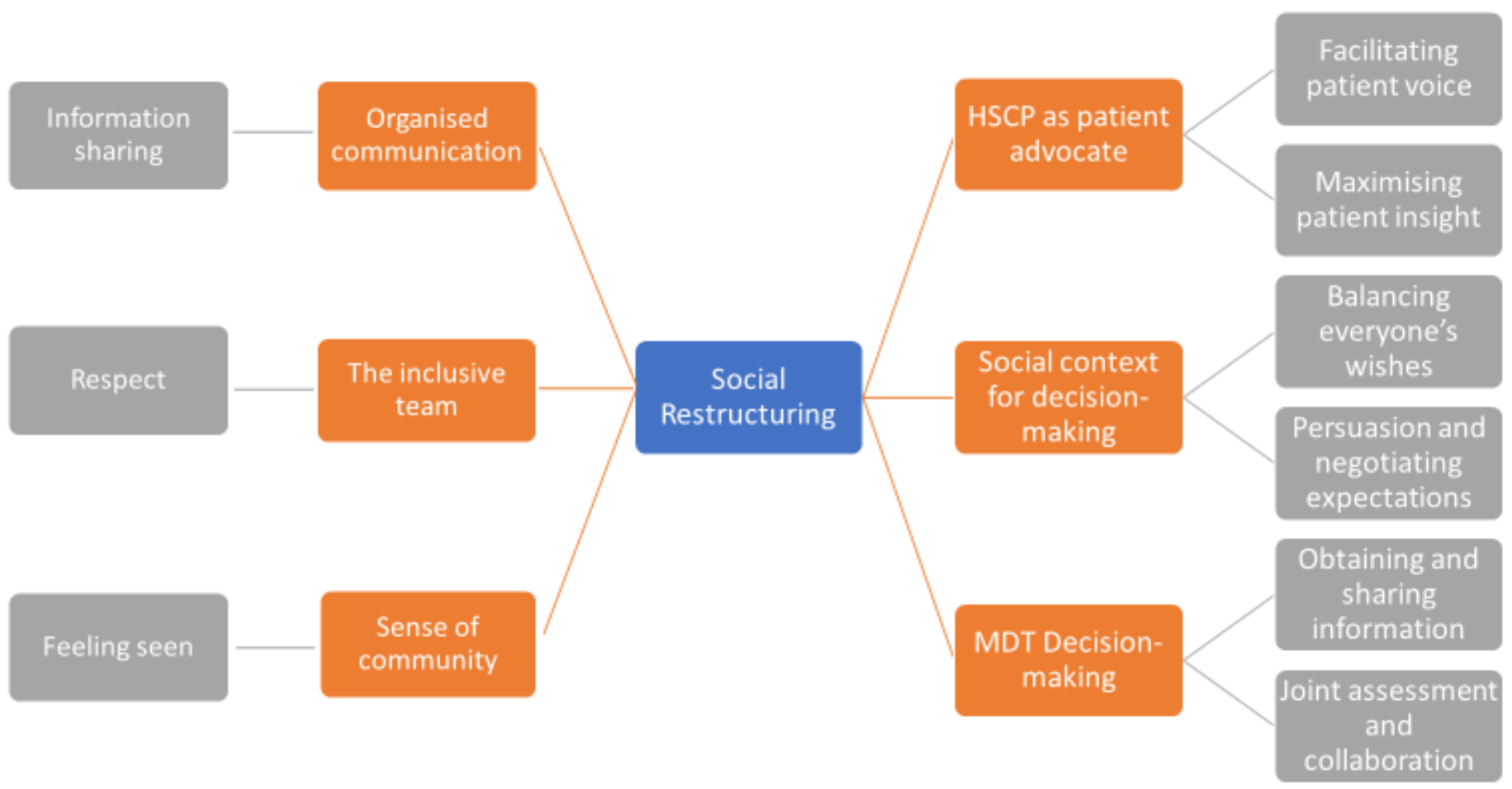

\section{Figure 4}

Overview of the thematic structure of participant data under the domain of social restructuring 
OLDER PEOPLE AND FAMILY CARERS

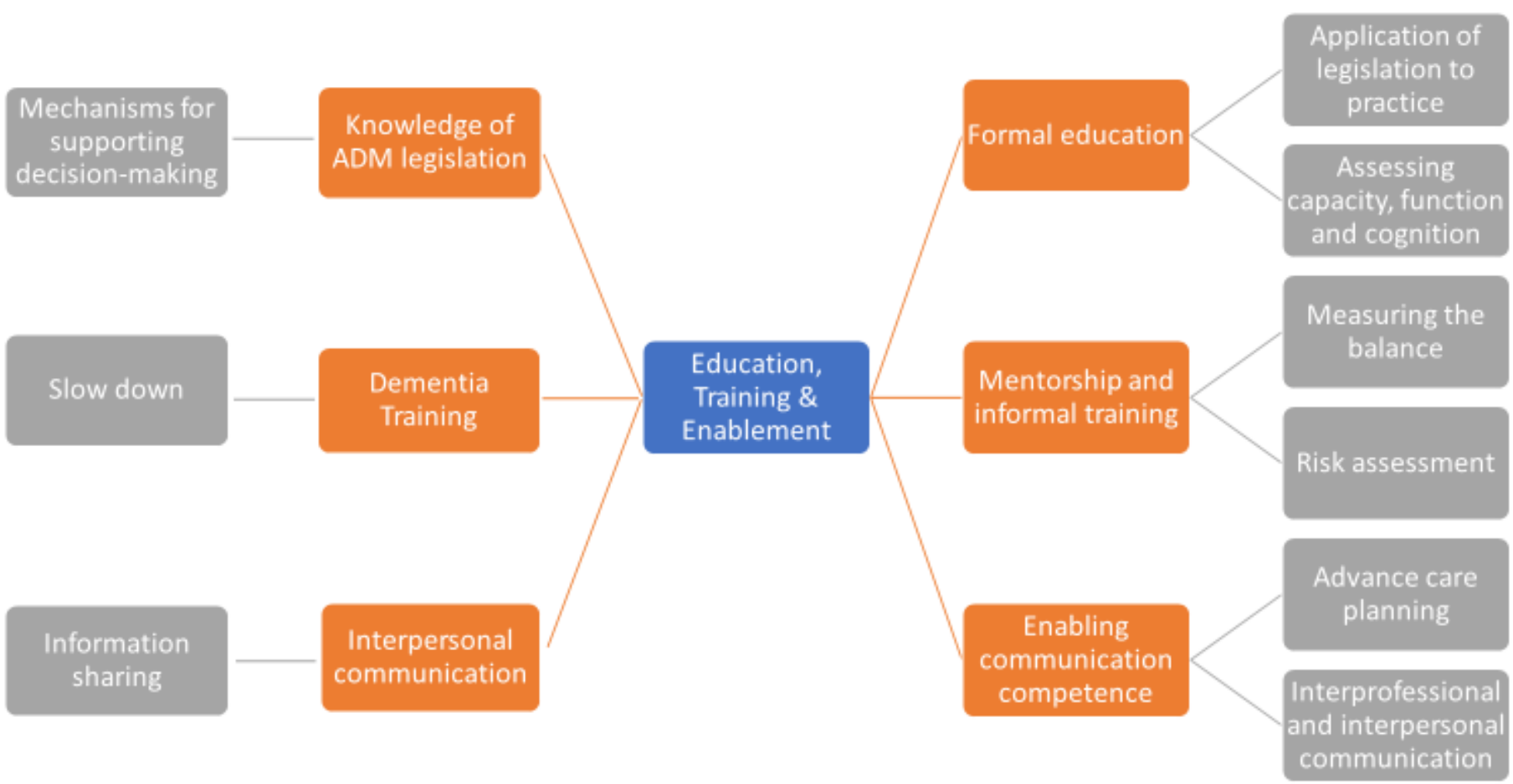

\section{Figure 5}

Overview of the thematic structure of participant data under the domain of education, training and enablement

\section{OLDER PEOPLE AND FAMILY CARERS}

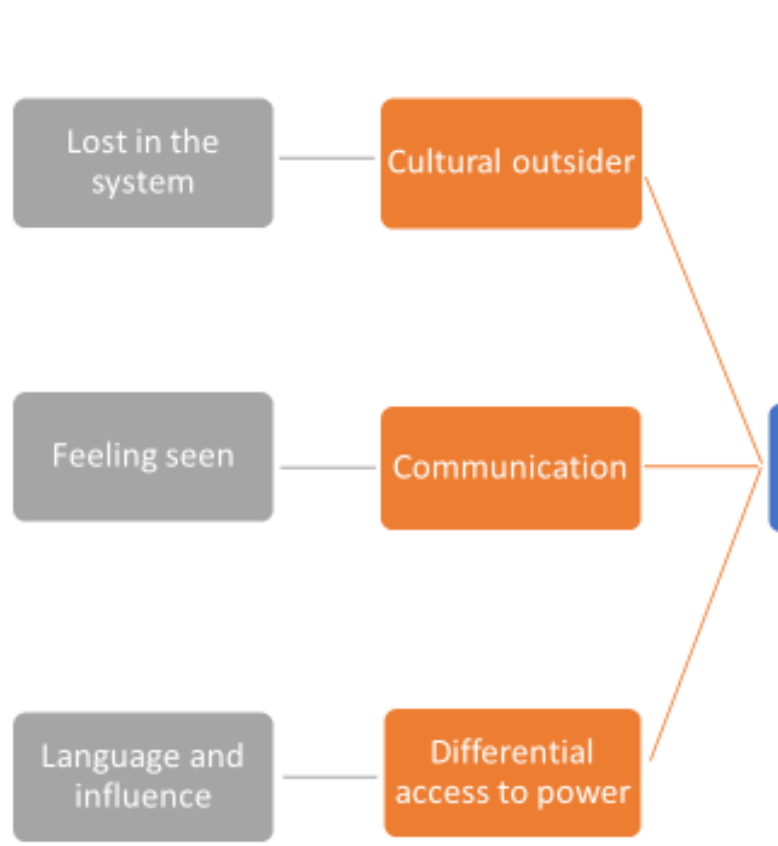

\section{HEALTH AND SOCIAL CARE PROFESSIONALS}

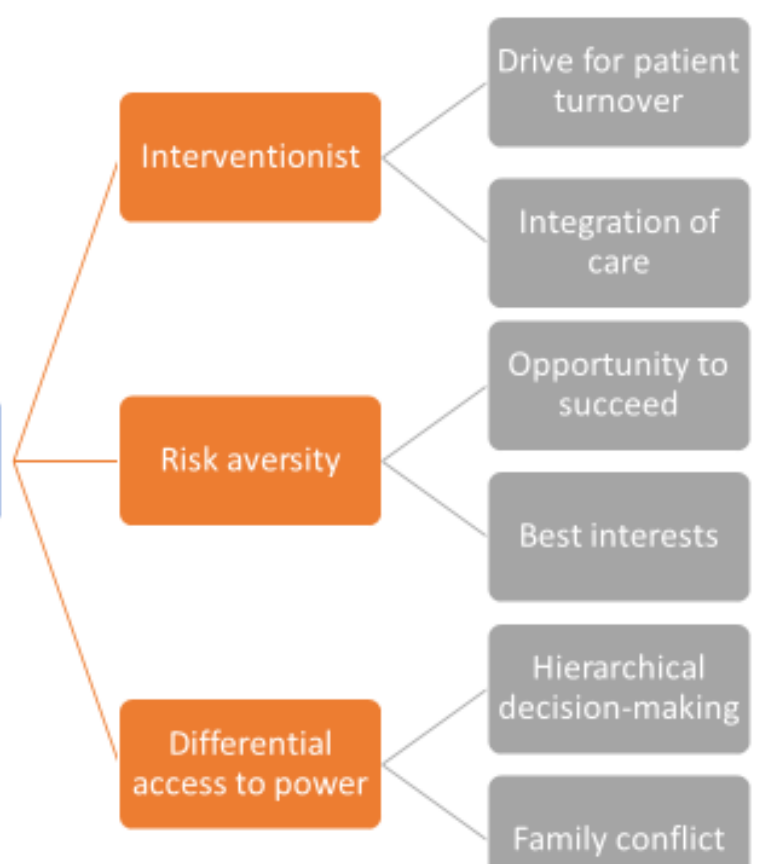


Figure 6

Overview of the thematic structure of participant data under the domain of culture and leadership 